\title{
RESEARCH HIGHLGHHS: Perovs kites
}

\author{
By Prachi Patel
}

Feature Editor: Pabitra K. Nayak

Research on perovskites has progressed rapidly, with new solar-cell efficiency records being set at a regular pace. Perovskite manufacturer Oxford PV announced in December a new record certified efficiency of $28 \%$ for their perovskite-silicon tandem solar cell. The first commercial products could reach the market by 2020, a decade since perovskite photovoltaics were first discovered. MRS Bulletin presents a selection of recent advances in this burgeoning field.

$\mathrm{R}$ esearchers have made an all-perovskite tandem solar cell with a record high power-conversion efficiency of $21 \%$.

Tandem solar cells are more efficient than a single cell because each device in the stack can be tailored to absorb a different part of the light spectrum. Tandem perovskite/silicon cells are closer to market, but all-perovskite tandem cells would be easier and less costly.

Making an all-perovskite tandem cell that is efficient has been a challenge. The bottom device in a tandem cell is prepared with a low-bandgap material to absorb all of the infrared photons passing through the top device. Despite many efforts, researchers have had difficulty making high-quality low-bandgap perovskite absorber layers.

Yanfa Yan, at The University of Toledo, and his colleagues made a highquality layer by introducing $2.5 \%$ chlorine into a mixed tin-lead perovskite. This increased grain size and crystallinity of the layer and reduced electronic disorder, which quashed the charge-carrier recombinations that produce heat and boost efficiency of the tandem cell. The cell reported in Nature Energy (doi:10.1038/

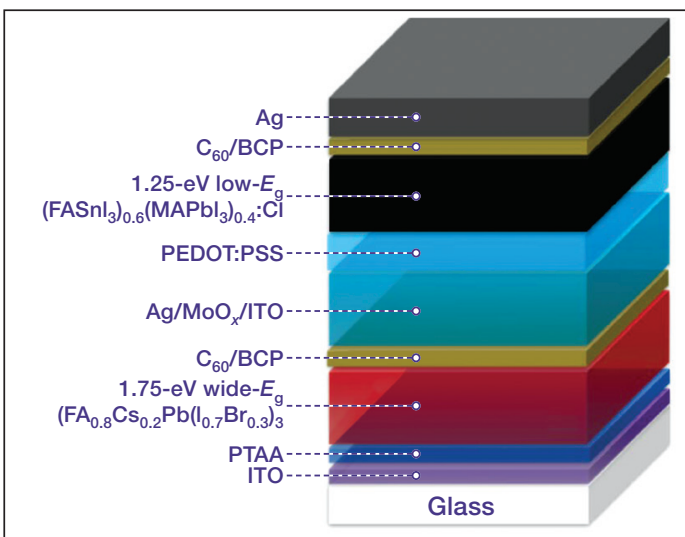

A schematic diagram of the $2 \mathrm{~T}$ tandem cell structure used in the study. Credit: Nature Energy.
$\mathrm{T}$ he presence of lead in state-of-the-art perovskite solar cells could hold back their commercialization. Lead-free alternatives based on tin compounds have shown promise, but they typically suffer from low efficiency and stability.

Brown University's Yuanyuan Zhou and Nitin Padture and their colleagues have made a "surprising discovery" that provides a solution. Simply adding germanium to the lead-free perovskite cesium tin iodide $\left(\mathrm{CsSnI}_{3}\right)$, which degrades easily, makes it air-tolerant, they found. Devices made with the new $\mathrm{CsSn}_{0.5} \mathrm{Ge}_{0.5} \mathrm{I}_{3}$ perovskite show an efficiency of $7.11 \%$ and remain highly stable after 500 hours $\mathrm{s} 41560-018-0278-\mathrm{x})$ retains $85 \%$ of this efficiency after 80 hours.
$\mathrm{M}$ etal-halide perovskite solar cells degrade when exposed to oxygen and moisture. Encapsulating the devices makes them more stable and long-lasting. But it does not solve one issue that crops up during regular device operation. Light, electric field, and thermal stress can all make lead and iodide ions more reactive, generating lead and iodine defects
$\left(\mathrm{Pb}^{0}\right.$ and $\left.\mathrm{I}^{0}\right)$ that serve as recombination centers for charge carriers and bring down device efficiency and lifetime.

Researchers at Peking University in Beijing have invented a novel technique for combating these defects. They added the rare-earth europium ion pair $\mathrm{Eu}^{3+}-\mathrm{Eu}^{2+}$ to lead-iodide perovskites. The redox pair shuttled electrons in a cyclical of operation under 1-sun illumination. The key to this behavior is the extremely high oxidation activity of Ge, which forms an ultrathin, uniform oxide layer on the surface, "which fully encapsulates and passivates the perovskite surfaces," the researchers write in their Nature Communications paper (doi:10.1038/s41467-018-07951-y). 
$\mathrm{W}$ hile lead-halide perovskites have revolutionized photovoltaics, they have also shown promise for lasers, light-emitting diodes, and transistors. Now, researchers show that perovskite nanocrystals are also highly effective catalysts for organic synthesis.

Reactions that form carbon-carbon bonds are the basis of synthesizing drugs, plastics, and chemicals. But the reaction procedures are complicated and require expensive noble metal catalysts. A team led by Yong Yan at San Diego State University found that colloids of methylammonium lead tribromide and cesium lead tribromide are 1000 times as effective as iridium- and ruthenium-based catalysts for catalyzing the $\alpha$-alkylation of aldehydes, a valuable and widely used chemical reaction. The perovskites cost approximately 100 times less. For the simple one-pot reaction, the researchers mixed organic starting materials into a suspension of the perovskite nanocrystals. Blue-light illumination triggers reactions that generate several products. By tweaking the reaction condition, the researchers can selectively catalyze other important chemical reactions, the researchers report in the Journal of the American Chemical Society (doi:10.1021/jacs.8b08720).
CoCrFeNi increases strength at cryogenic temperatures

$\mathrm{A}^{\mathrm{n}}$ international team of researchers has discovered that the strength of a CoCrFeNi high-entropy alloy increases at the liquid-helium temperature while the sample maintains excellent ductility. These mechanical properties could make this new alloy useful for cryogenic applications, according to the study published recently in Science China Materials (doi:10.1007/s40843-018-9373-y).

The CoCrFeNi alloy belongs to an emerging class of materials known as high-entropy alloys (HEAs). Compared to traditional alloys such as steel, which mostly consist of one primary metal with small concentrations of additional elements, HEAs are mixtures of several different elements in roughly equal concentrations. HEA research was ignited in the early 2000s with the simultaneous discovery of two different alloys. Today, research continues on HEAs in particular because they exhibit desirable mechanical and structural properties at extreme temperatures. For example, "HEAs usually have different kinds of defects [compared to conventional alloys], such as stacking faults with lower energy, and can be ductile even at very low temperatures," says Yong Zhang of the University of Science and Technology Beijing. Zhang is corresponding author of the publication.

Zhang and his collaborators performed a series of challenging experiments on $\mathrm{CoCrFeNi}$, assessing the alloy's strength and ductility down to temperatures of $4.2 \mathrm{~K}$. "Few places in the world can per- form these extremely low temperature experiments," says Peter Liaw of The University of Tennessee, Knoxville, who is a coauthor on this publication. When subjected to tensile strength testing at extremely low temperatures, the $\mathrm{CoCrFe}$ $\mathrm{Ni}$ alloy deformed in a slip-stick manner, as demonstrated by jagged or serrated stress-strain curves at $20 \mathrm{~K}$ and $4.2 \mathrm{~K}$.

Performing mechanical property measurements at "the liquidhelium temperature is really remarkable," says Richard LeSar of Iowa State University, "It opens up a different realm for thinking about behaviors in these systems." LeSar was not involved with this study.

To explain the serrated behavior of the stress-strain curves, images of the $\mathrm{CoCrFeNi}$ samples after strain testing were taken using both a transmission electron microscope and a high-resolution scanning transmission electron microscope. The images revealed many small parallel hatched features consistent with the formation of deformation twins, which correspond to boundaries where two different regions of the alloy have shifted
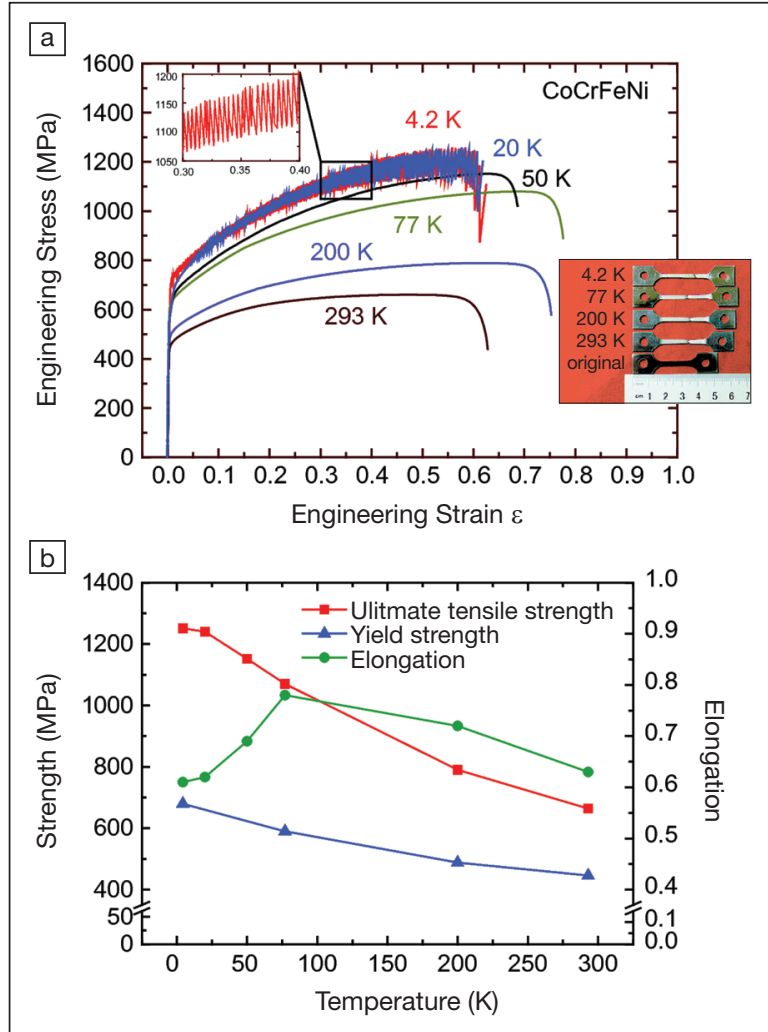

Tensile properties of the CoCrFeNi alloy at different temperatures. (a) Engineering stress-strain curves and photograph of the dogbone-shaped samples, before and after tensile tests. The enlarged stress-strain curve at $4.2 \mathrm{~K}$ is displayed in the inset and the jagged serrations are clearly observed. (b) Strength and elongation versus temperature for the high-entropy alloy. The ductility reaches a maximum at 77 K. Credit: Science China Materials. 